\title{
STATE EQUATION APPROXIMATION OF TRANSFER MATRICES AND ITS APPLICATION TO THE PHASE DOMAIN CALCULATION OF ELECTROMAGNETIC TRANSIENTS
}

\author{
A. Oguz Soysal* Adam Semlyen
}

Department of Electrical Engineering

Toronto, Ontario, Canada, M5S 1A4

- On leave from Karadeniz Technical University, Trabzon, Turkey

Abstract - A general methodology is presented for the state equation approximation of a multiple input-output linear system from transfer matrix data. A complex transformation matrix, obtained by eigenanalysis at a fixed frequency, is used for diagonalization of the transfer matrix over the whole frequency range. A scalar estimation procedure is applied for identification of the modal transfer functions. The state equations in the original coordinates are obtained by inverse transformation. An iterative Gauss-Newton refinement process is used to reduce the overall error of the approximation.

The developed methodology is applied to the phase domain modeling of untransposed transmission lines. The approach makes it possible to perform EMTP calculations directly in the phase domain. This results in conceptual simplification and savings in computation time since modal transformations are not needed in the sequences of the transient analysis. The presented procedure is compared with the conventional modal approach in terms of accuracy and computation time.

Keywords: Transfer function matrices, Parameter estimation, Frequency response, Identification, State equation approximation, Electromagnetic transients.

\section{INTRODUCTION}

Modeling of power system components has been a major concern in the development of the computational tools for electromagnetic transient studies. At present, the commonly used programs based on the procedure described in [1] and [2] perform the simulations in the time domain because of the resulting flexibility in simulating switching operations and fault conditions. However, the modeling of various components has initially to be made with frequency domain formulation in order to reflect their frequency dependent behavior.

The transmission line parameters can be determined by using basic field relations together with Carson's formulae or with the concept of complex penetration depth. The frequency domain behavior of a transmission line is usually characterized by two complex functions, the propagation transfer function $H_{P}$, and the characteristic admittance (or impedance) function $Y_{C}\left(Z_{C}\right)$. The line models used in EMTP (the Electro-Magnetic Transients Program) are based on the rational function approximation of the wide frequency range variation of the $H_{P}$ and $Y_{C}$ functions [3]-[5].

In conventional EMTP studies modal transformations are used for modeling of unbalanced multiphase lines. Calculations related to raveling waves are performed for the decoupled modes and phase domain variables are obtained by inverse transformation. It has been common practice to use constant real transformation matrices for modal decomposition. Because of frequency dependence, a fixed transformation can obviously not provide perfect diagonalization over a wide frequency range. In most cases, where the frequency dependence is not too significant, the non-diagonal elements of the transformed $H_{P}$ and $Y_{C}$ matrices may be neglected, so that the modes can be assumed decoupled.

93 WM 240-2 PWRS A paper recommended and approved by the IEEE Power System Engineering Committee of the IEEE Power Engineering Society for presentation at the IEEE/PES 1993 Winter Meeting, Columbus, OH, January 31 - February 5, 1993 . Manuscript submitted August 10, 1992; made avallable for printing December 28, 1992.
Transformers are also essential components of power systems. Various transformer models have been proposed for the analysis of electromagnetic transients. Either detniled winding models or external terminal representations may be used depending on the availability of the required data [6]-[9]. A general survey of the literature indicates, however, the need for an improved frequency dependent representation of three phase multi-winding transformers applicable to standard EMTP studies [10]. Thus, the need for solving the general problem of multi-terminal analysis for different system components or multi-port network equivalents [11] becomes apparent.

In this paper, a general methodology is presented for the state space modeling of multiple input-output linear systems from frequency domain transfer matrix data. It is applicable in any relevant field of dynamic systems analysis. Either a measured or an analytically obtained frequency response matrix may be used as a data base for describing the process. A complex transformation matrix obtained by eigenanalysis at a fxed frequency is used for diagonalization of the transfer matrix over the whole frequency range. A scalar estimation procedure [9] is used to identify the modal transfer functions. The transfer matrix in the original coordinates is then approximated by state equations by an iterative process (a Gauss-Newton SVD refinement). The state equation realization of a transfer matrix, as shown in this paper, is a minimal realization [12] as its order corresponds to that required by the diagonalized matrix. Therefore, it is of much lower order than what could be obtained by "brute force" through fitting to each element of the matrix. Still, diagonalizability of the transfer matrix (by a constant transformation) is not a requirement due to the final refinement procedure.

The particular objective of the paper is the application of the presented methodology to the phase domain modeling of untransposed transmission lines. The approach makes it possible to perform the EMTP calculations directly in the phase domain rather than in the modal domain. The presented procedure is compared with the conventional modal approach in terms of accuracy and computation time. While both methods provide good accuracy, the direct phase domain approach can serve as a standard of reference and also results in reduced computation time. Although the specific application shown is for overhead transmission lines, as a first test of feasibility, the methodology is general and can be used for other power system equipment such as three phase transformers, rotating machines, and cables. In the latter case, to be examined in a sequel to this paper, its use seems to be almost unavoidable because of the strong frequency dependence of the modal transformation matrices [13].

\section{STATE EQUATION APPROXIMATION OF TRANSFER MATRICES}

The outline of the basic methodology developed for the state equation approximation of power system components is presented below. The approach may be applied to any multiple input-output system for which frequency response data are known in a certain frequency range. The particular application to the modeling of transmission lines for electromagnetic transient analysis is given in the next section.

\section{Methodology}

Suppose that the frequency response of a multiple input-output system is given as

$$
\mathbf{y}=\mathbf{H u}
$$

In the general case $\mathbf{H}$ is a symmetrical or non-symmetrical complex matrix. While the number of the inpuls and outputs may be different, we assume, for the purpose of this paper, that the system has $r$ inputs and $r$ outputs. $u$ and $y$ are complex vectors, designating the input and output variables respectively. We assume that the transfer marix $\mathbf{H}$ is 
known for $m$ discrete observation frequencies, $\omega_{k}$, in the interval $\omega_{a} \leq \omega_{k} \leq \omega_{b}$.

Diagonalization

Let us define the linear transformations to modal variables

$$
\mathbf{u}=\mathbf{T}_{\mathbf{u}} \mathbf{u}_{m} \quad \mathbf{y}=\mathbf{T}_{\mathbf{m}} \mathbf{y}_{\mathbf{m}}
$$

Substituting (2) into (1) yields

$$
\mathbf{y}_{m}=\mathbf{H}_{m} \mathbf{u}_{m}
$$

where

$$
\mathbf{H}_{m}=\mathbf{T}_{\mathbf{y}}^{-1} \mathrm{HT}_{\mathbf{v}}
$$

The transformation matrices can be determined in such a way that $\mathbf{H}_{m}$ is diagonal for a particular frequency $\omega_{0}$. In particular, the eigenvector matrix $T_{0}$ of $H$, evaluated at the selected frequency $\omega_{0}$, may be used for diagonalization by setting $T_{u}=T_{y}=T_{0}$. In the general case, however, different transformation matrices $T_{30}$ and $T_{40}$ are used for the input and output variables if they are of different physical nature (e.g., voltage, current).

If $\mathrm{H}$ is the frequency response of a component involving frequency dependent parameters, it may not be possible to find a fixed transformation to diagonalize $H$ over the whole frequency range. The use of a frequency dependent transformation is, however, in general not practical since it has to be used for time domain simulations. A number of real constant transformations which diagonalize a balanced matrix have been used in some particular applications [2]. For the general case of unbalanced systems, it has been common to perform the eigenanalysis at a fixed transformation frequency $\omega_{0}$ to obtain the transformation matrices to be used over the complete frequency range. Studies related to the frequency dependence of the modal transformation (e.g. [14] and [15]) have shown that reasonably accurate results can be obtained in some practical cases by a particular selection of the transformation frequency. At frequency values other than $\omega_{0}$ the non-diagonal elements of $\mathbf{H}_{m}$ are not zero but are, in general, small compared to the diagonal elements. We assume here for generality that the non-diagonal elements of $\mathbf{H}_{m}$ are not negligible, but in order to perform the initial estimation we define the approximate frequency response by considering only the diagonal elements.

\section{Initial state equation approximation}

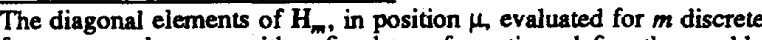
frequency values $\omega_{k}$ with a fixed transformation, define the weakly coupled modal (scalar) transfer functions

$$
\begin{gathered}
H_{\mu}\left(j \omega_{k}\right)=\left[\mathbf{H}_{m}\left(j \omega_{k}\right)\right]_{\mu, \mu} \\
\left(\omega_{a} \leq \omega_{k} \leq \omega_{b} \quad \mu=1, \ldots, r \quad k=1, \ldots, m\right)
\end{gathered}
$$

In order to obtain an initial state equation approximation, rational functions are fitted separately to the modal frequency responses $H_{\mu}$ by using the scalar estimation process described in [9]. The methodology is based on the identification of the pole locations and strengths (residues) by the solution of a least squares problem using singular value decomposition. The process includes also an iterative improvement stage with direct restriction on the real parts of the poles to impede obtaining unstable poles. The estimated modal transfer functions can be written as

$$
H_{\mu}(s)=\sum_{i} \frac{\alpha_{\mu, i}}{s-\lambda_{\mu, i}} \quad\left(\mu=1, \ldots, r \quad i=1, \ldots, n_{\mu}\right)
$$

In the general case, $\alpha_{\mu, i}$ and $\lambda_{\mu, i}$ (designating the strength and location of poles) may be real and/or complex (conjugate) numbers. Here all poles will be assumed to be complex conjugate to simplify the notation. Real poles can also be considered as complex conjugate with zero imaginary part, by splitting the corresponding pole strength to two equal parts. $H_{\mu}(s)$ is assumed to be strictly proper (i.e. $\lim H_{\mu}(s)=0$ for $\left.s \rightarrow \infty\right)$. This assumption does not introduce any restriction, since in the case of a proper transfer function, the problem can be reduced to the strictly proper case by subtracting from $H_{\mu}(s)$ its value for $s \rightarrow \infty$.

The modal transfer functions obtained are equivalent in the time domain to the state equations

$$
\begin{aligned}
& \dot{\mathbf{x}}(t)=\Lambda \mathbf{x}(t)+\mathrm{B}_{m} \mathrm{u}_{m}(t) \\
& \mathbf{y}_{m}(t)=\mathbf{C}_{m} \mathbf{x}(t)
\end{aligned}
$$

where

$$
\begin{array}{lll}
\Lambda=\operatorname{diag}\left(\Lambda_{\mu}\right\} & B_{m}=\operatorname{diag}\left(b_{m, \mu}\right) & C_{m}=\operatorname{diag}\left\{c_{m, \mu}^{T}\right\} \\
\Lambda_{\mu}=\operatorname{diag}\left(\lambda_{\mu, i}\right) & b_{m, \mu}=\operatorname{col}\left(\alpha_{\mu, i}\right) & c_{m, \mu}=\operatorname{col}(1)
\end{array}
$$

$$
\left(i=1, \ldots, n_{\mu} \quad \mu=1, \ldots, r\right)
$$

The original variables, $u$ and $y$, can be substituted into the state equations (7) by using the inverse of the transformations defined in (2) at the selected frequency $\omega_{0}$. This yields

$$
\begin{gathered}
\dot{\mathbf{x}}(t)=\mathbf{A x}(t)+\mathrm{B}_{0} \mathrm{u}(t) \\
\mathbf{y}(t)=\mathrm{C}_{0} \mathbf{x}(t) \\
\mathrm{B}_{0}=\mathbf{B}_{\mathrm{m}} \mathbf{T}_{=0}^{-1} \quad \mathrm{C}_{0}=\mathbf{T}_{y 0} \mathrm{C}_{\mathrm{m}}
\end{gathered}
$$

\section{Balancing of the complex rows and colunns}

In the case of complex conjugate poles, the corresponding rows and columns in the $B_{m}$ and $C_{m}$ matrices are also complex conjugate. However, the multiplication with the transformation matrices to obtain the original variables changes this structure in $\mathbf{B}_{0}$ and $\mathbf{C}_{0}$, so that their complex columns or rows, respectively, are not conjugate pairs, which makes it impossible to find an equivalent state model with real parameters. Therefore, before starting the iterative process, it is necessary to balance the matrices $B$ and $C$ in such a way that the rows and columns corresponding to complex conjugate poles remain complex conjugate.

Let $\lambda_{i}$ and $\lambda_{j}$ be two complex conjugate poles $\left(\lambda_{j}=\lambda_{i}\right)$. We designate the corresponding rows and columns of the $B_{0}$ and $C_{0}$ matrices which are not conjugate pairs by $b_{i}^{T}, b_{j}^{T}, c_{i}$ and $c_{j}$. The balanced rows and columns are obtained as

$$
\begin{array}{ll}
\overline{\mathbf{b}}_{i}=\frac{1}{2}\left(\mathbf{b}_{i}+b_{j}^{*}\right) & \overline{\mathbf{b}}_{j}=\overline{\mathbf{b}}_{i}^{*} \\
\overline{\mathbf{c}}_{i}=\frac{1}{2}\left(\mathbf{c}_{i}+\mathbf{c}_{j}^{*}\right) & \overline{\mathbf{c}}_{j}=\overline{\mathbf{c}}_{i}^{*}
\end{array}
$$

The balanced input and ouput matrices will be denoted by $\overline{\mathbf{B}}_{0}$ and $\overline{\mathbf{C}}_{0}$. Gauss-Newton refinement

The transfer matrix corresponding to the state equations (8)

$$
\mathbf{H}_{0}=\overline{\mathbf{C}}_{0}[s I-\mathbf{\Lambda}]^{-1} \overline{\mathbf{B}}_{0}
$$

is expected to fit the given frequency response $H_{k}$ for $s=j \omega$ $\left(\omega_{a} \leq \omega_{k} \leq \omega_{b}\right)$. In practice, the following factors are the principal sources of error in this fitting :

- The error in the scalar fitting

- Omission of the non-diagonal elements of $\mathbf{H}_{n}$

- Balancing of the rows and columns of $B_{0}$ and $C_{0}$

An iterative improvement process, termed Gauss-Newton SVD refinement, is developed for better approximation of the state equation parameters to reproduce the original frequency response more accurately. The elements of the transfer matrix obtained throughout the refinement steps can be written explicitly as

$$
H_{i, j}=\sum_{v} \frac{\bar{c}_{i, v} \bar{b}_{v, j}}{s-\lambda_{v}} \quad(i=1, \ldots, r \quad j=1, \ldots, r \quad v=1, \ldots, n)
$$

where $n$ is the total number of poles identified for all modal transfer functions. The parameters, assumed to be complex for generality, are defined as

$$
\lambda_{v}=\lambda_{v}^{\prime}+j \lambda^{\prime \prime} \quad \bar{b}_{v, j}=\bar{b}_{v, j}^{\prime}+j \bar{b}^{\prime \prime}{ }_{v, j} \quad \bar{c}_{i, v}=\bar{c}_{i, v}^{\prime}+\bar{j}^{\prime \prime}{ }_{i, v}
$$

$\lambda_{v}, \bar{b}_{v, j}$, and $\bar{c}_{i, v}$ are the variables of the Gauss-Newton SVD procedure. In the case of large deviations between the given frequency response and the initial estimation, convergence problems may occur in the iterative improvement process. The continuation method (see, for example, [16]), described below, can then be used to assure 
convergence.

Let us define an intermediate target transfer matrix $\mathrm{H}_{0}$ between the given frequency response $H$ and the initial phase domain transfer matrix $H_{0}$ of (10) by introducing an arbitrary parameter $\theta$ :

$$
\mathrm{H}_{\theta}=(1-\theta) \mathrm{H}_{0}+\theta H
$$

The parameter $\theta$ is varied from 0 to 1 with increments small enough to assure convergence. The problem is, therefore, to minimize the difference between the estimated transfer matrix and the intermediate target transfer matrix $\mathrm{H}_{\theta}$

$$
\overline{\mathbf{C}}\left[j \omega_{k} \mathrm{I}-\mathbf{\Lambda}\right]^{-1} \overline{\mathbf{B}}-\mathbf{H}_{\theta}\left(j \omega_{k}\right) \rightarrow 0 \quad(k=1, \ldots, m)
$$

by adjusting the coefficient matrices $\Lambda, \overline{\mathbf{B}}$, and $\overline{\mathbf{C}}$. For each $\theta$ GaussNewton iterations, as shown below, are applied to update the parameters until the euclidian norm of the left side of (14) becomes smaller than a predefined tolerance. At any iteration step, the deviation (14) from the target frequency response can be expressed as

$$
\begin{aligned}
& f(\xi)=\mathrm{f}+\mathrm{j} \mathrm{f}^{\prime}=\operatorname{col}\left\{H_{i j}\left(\xi, j \omega_{k}\right)-H_{\theta, i j}\left(j \omega_{k}\right)\right\} \\
& (i=1, \ldots, r \quad j=1, \ldots, r \quad k=1, \ldots, m)
\end{aligned}
$$

where $\xi$ is the real vector of variables containing the real and imaginary parts of the state equation parameters in $\mathbf{A}, \vec{B}$, and $\bar{C}$ of (14):

$$
\xi=\left[\xi_{\lambda}, \xi_{b}, \xi_{c}\right]^{T}
$$

$\xi_{2}=\left[\cdots \lambda_{i}^{\prime}, \lambda^{\prime \prime} \cdots\right] \quad \xi_{b}=\left[\cdots \bar{b}_{i j}^{\prime}, \bar{b}^{\prime \prime}{ }_{i j} \cdots\right] \quad \xi_{c}=\left[\cdots \bar{c}_{j i}^{\prime}, \bar{c}^{\prime \prime}{ }_{j i} \cdots\right]$

Equating the deviation to zero, we obtain a set of overdetermined nonlinear equations, which can be linearized by Newton's method and solved using a least squares routine. Due to the linearized least squares nature of the problem, the process is referred to as GaussNewton improvement. We have thus

$$
J_{\text {old }}\left(\xi-\xi_{\text {old }}\right)=-f\left(\xi_{\text {odd }}\right)
$$

where

$$
J_{P q}=J_{p q}^{\prime}+j^{\prime \prime}=\frac{\partial f_{p q}(\xi)}{\partial \xi_{q}} \quad(p=1, \ldots, M \quad q=1, \ldots, N)
$$

$J$ is the $M \times N$ complex jacobian matrix. $M=m r^{2}$ is the dimension of the vector $f$. The total number of parameters is $N_{1} \equiv(2 r+1) n$. However, $n$ paramaters related to either the $\bar{B}$ or the $\bar{C}$ matrix may be chosen arbitrarily for normalization. Besides, the imaginary parts of the parameters known to be real should not take place in the vector $\xi$, since they are identically equal to zero. The number of the unknown parameters is thus $N=2 r n-n_{r e}$, where $n_{r e}$ is the number of real poles. To reduce the problem to the solution of real linear equations, we define the new set of variables

$$
\Delta \xi=\xi-\xi_{\text {old }}
$$

and separate the real and imaginary parts of (17):

$$
\Psi \Delta \xi=\Phi \quad \Psi=\left[\begin{array}{l}
J^{\prime} \\
J^{\prime}
\end{array}\right] \quad \Phi=-\left[\begin{array}{l}
f \\
\mathbf{f}
\end{array}\right]
$$

The overdetermined equation (20) can be solved by singular value decomposition (SVD) with the inequality constraints $\lambda^{\prime} \leq 0$ on the real parts of the poles, to assure stability at each step.

The continuation method has been given here for generality and completeness. The approach is needed especially if the error in the initial fitting is large. However, it should be noted that in some applications, satisfactory convergence may be obtained for $\theta=1$, which is equivalent to by-passing the continuation loop.

We note that matrix $H(s)=\bar{C}[s I-\Lambda]^{-1} \bar{B}$, resulting from the refinement process described above, has eigenvectors that are functions of $s$ and, consequently, the modal transformation matrices are frequency dependent, despite the fact that $\bar{B}$ and $\bar{C}$ are constant. Thus the initial diagonalizability of the transfer matrix does not exist in the end result.

The state equations obtained through the Gauss-Newton refinement contain in general both real and complex conjugate parameters. In practice, however it is more convenient to use real models.
A number of equivalent real representations can be found by simple algebraic expressions. The formulation used in this work is described in Appendix A. With this particular formulation, the state equations are directly obtained in standard form, with real coefficient matrices:

$$
\begin{aligned}
& \dot{\mathbf{x}}(t)=\mathbf{A x}(t)+\mathbf{B u}(t) \\
& \mathbf{y}(t)=\mathbf{C x}
\end{aligned}
$$

\section{Partitioning}

Equation (20) represents a set of $2 M$ linear equations with $N$ unknowns. Both $M$ and $N$ depend on the total number of poles, the number of inputs and outputs, and the number of observations. In the case of many input-output systems the size of the jacobian matrix can be very large, especially if the frequency response is oscillatory, when a large number of observations becomes necessary to obtain satisfactory fitting. In such cases, an appropriate partitioning of the problem is needed to reduce both the storage space and the computation time.

In the case of an oscillatory frequency response, the euclidian norm $\|\mathrm{H}\|$ may have many minima and maxima over the frequency range of the given observation data. Let us partition this frequency range into reasonable intervals considering the essential minima of $\|\mathbf{H}\|:$

$$
\Omega=\left\{\omega_{a}, \ldots, \omega_{b}\right\}=\left\{\Omega_{p}\right\} \quad\left(p=1, \ldots, n_{p}\right)
$$

Here $\Omega_{p}$ is a subset of $\Omega$, containing contiguous frequencies. The full set $\Omega$ of frequencies is the union of the ordered subsets $\Omega_{p}$. Equation (14) can be partitioned by focusing on the segment $\Omega_{p}$ of the frequency scale:

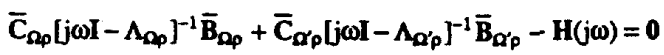

where $\Omega_{p}^{\prime}$ designates the complement of $\Omega_{p}$, with respect to the full range $\Omega$ :

$$
\Omega_{p}^{\prime} \cup \Omega_{p}=\Omega
$$

By defining

$$
\mathbf{H}_{\boldsymbol{\gamma}_{\varphi}}(j \omega)=\mathbf{H}(j \omega)-\overline{\mathbf{C}}_{\boldsymbol{\Omega}_{p}^{\prime}}\left[j \omega I-\Lambda_{\boldsymbol{\alpha}_{p}^{\prime}}\right]^{-1} \overline{\mathbf{B}}_{\boldsymbol{\alpha}_{p}}
$$

we can write

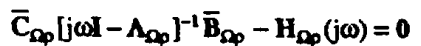

The Gauss-Newton refinement described in $(17)-(20)$ can now be applied to (25). The parameters are identified section by section, by varying $p$ from 1 to $n_{p}$. The iterations continue until the predefined error level is satisfied.

\section{Algorithm}

The estimation process described above, can be summarized by the following algorithm:

Input : $\mathbf{H}_{k}$, transfer matrix given for $m$ discrete observation frequencies, $\omega_{a} \leq \omega_{k} \leq \omega_{b}$.

1 - Define the transformation frequency $\omega_{0}$, determine the transformation matrices $T_{k 0}, T_{y 0}$ and compute the modal transfer functions by using ( 3 ) for $k=1, \ldots, m$.

2 - Run the scalar estimation routine for each of the modal transfer functions.

3 - Perform the inverse transformation according to (8), rearrange the state equations in such a way that the poles are sorted according to their imaginary parts. Form the vector of parameters as defined in (16).

4 - If the continuation method is needed, choose the increment $\Delta \theta$, set $\theta=0$. (If convergence can be obtained without the continuation method, $\Delta \theta=1$ )

5 - Determine the minima of $\left\|H_{k}\right\|(k=1, \ldots, m)$ and define $\Omega_{p}$ $\left(p=1, \ldots, n_{p}\right)$. Partition the poles according to their imaginary parts. (If partitioning is not needed, $n_{p}=1$ )

6 - Set $\theta=\theta+\Delta \theta$, compute the intermediate target function as per (13).

7 - Update the elements of $\Lambda_{\Omega_{p}}, \overline{\mathbf{B}}_{\rho_{p}}$, and $\overline{\mathbf{C}}_{\rho_{p}}$ for $p=1, \ldots, n_{p}$. using the Gauss-Newton refinement described in (15)-(20). Check \|f\|, if greater than the predefined error limit, repeat this 
tep.

8 - If $\theta<1.80$ to 6.

9 - Extimation of the complex parameters is completed. Obtain the real thate oquations with real parameters (21) using the exprescioms given in Appendix A.

\section{Exonmple}

As a particular application, the methodology described above is used for the state equation approximation of tranamiusion line transfer matrices. As shown in the next section. the frequency domain behavior of a transmission line is charactorized in EMTP studies by the propagation and the characteristic admittance (or impedance) matrices. Application of the proposed procedure to three different line arrangements specified in Appendix B resulted in state equation pproximetion of both tran fer matrices with an overall error in the order of 1\%. Line-2 appears to be the mont wevere case for the process, due to the unbalnoce of the parameters. Some details of the state equation approximation of the propagation transfer matrix of Line-2 are presented below.

The froquency response to be used as input data is obtained from the geometric dimencions of Line-2 given in Appendix B. The

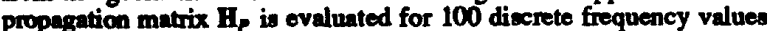
equally epaced along the logarithmic acale, in the range $1 \mathrm{~Hz} S f \leq 1 \mathrm{MHz}$, with line parameters determined using the concept of the complex penetration depth. The seloction of the transformation frequency is not as important as in the conventional modal approach. since the Gaum-Newton refinement process efficaciously improves the final epproximation. However, an appropriate choice of this frequency reduces the computation time required for the Gauss-Newton refinement. The diagonalization is performed with a transformation matrix obtained at $f_{0}=35 \mathrm{kHz}$. The remulting modal transfer functions are thown in Figure 1. The mummary of the scalar estimation is presented in Trble 1 .

The error levels shown in Table 1 are the RMS of the absolute values of the complex differences betwoen the input data and the extimated rational function. This encompacses both the errors of magnitude and angle.

The atate equations obtained by inverse tranuformation lead to an overall RMS error of $6.16 \%$. Three steps of Gauss-Newton refinement reduced the error to $1.12 \%$. The resulting fitting plotted for one diagonal and one off-diagonal element of the propagation matrix $\mathbf{H}_{\text {, }}$ is shown in Figure 2. The error of less than $1 \%$ in the lower frequency range can of course be further reduced by a small increase of the number of poles.

The example of atate equation approximation presented above wes performed with a MIPS-RISC 6280 computer. CPU times for different stages of the process are:

- Dingonalization with modal transformation : $0.18 \mathrm{~s}$

- Scalar extimation for six modes : $1.18 \mathrm{~s}$

- Gaves-Newton refinement : 19.83 s

The CPU time needed for the Gause-Newton refinement is conviderably higher than for the rest of the approximation process. It is, however, not excessive. The number of the refinement steps may be reduced or the refinement may be skipped if a higher error level is acceptable.

\section{PHASE DOMAIN MODELING OF} TRANBMISSION LINE TRANSIENTS

A particular application of the presented general methodology is given here for the phase domain modeling of tranomivsion line tranvients. The accurate and efficient modeling of three phase lines, with due consideration of the frequency dependence of their parameters, has been the object of much effort in the EMTP development almost from its beginning.

EMTP is a time domain program based on the step by step integration of the ordinary differential equations (ODEs) representing the system components. Its efficiency is due to the computational decoupling between components separated by transmission lines, due to the delay time of the traveling waves. Direct time domain simulation also permits the representation of awitches in a straightforward way. However, basic tranamission line modeling is always in the frequency domain vince complex feld phenomena in the ground and inside the conductor result in parameters which are functions of frequency. The time domain representation of transmission lines requires

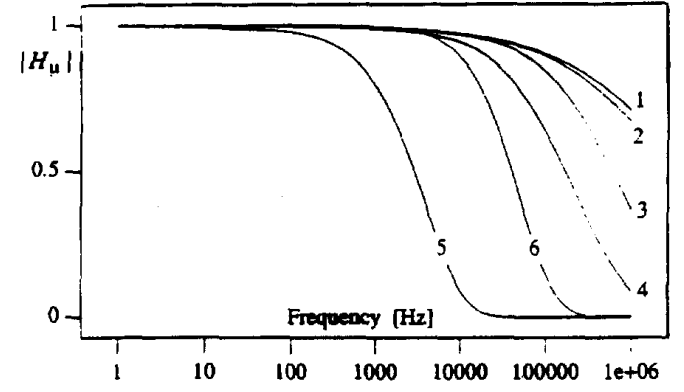

Figure 1 Modal propagation transfer functions for Line-2. $150 \mathrm{~km}$.

Table 1 Results of the scalar estimution of modal transfer functions

\begin{tabular}{|c|c|c|c|}
\hline Mode & Poles & Residues & Error (\%) \\
\hline 1 & $\begin{array}{l}-0.3933 \times 10^{8} \\
-0.8623 \times 10^{7} \\
-0.2139 \times 10^{7} \\
-0.2785 \times 10^{6}\end{array}$ & $\begin{array}{l}-0.1969 \times 10^{2} \\
-0.2169 \times 10^{7} \\
-0.2828 \times 10^{6} \\
-0.2893 \times 10^{5}\end{array}$ & 1.00 \\
\hline 2 & $\begin{array}{l}-0.3200 \times 10^{7} \\
-0.8545 \times 10^{7} \\
-0.2052 \times 10^{7} \\
-0.2722 \times 10^{6}\end{array}$ & $\begin{array}{l}-0.1326 \times 10^{7} \\
-0.2571 \times 10^{7} \\
-0.3309 \times 10^{6} \\
-0.3034 \times 10^{5}\end{array}$ & 1.01 \\
\hline 3 & $\begin{array}{l}-0.2439 \times 10^{8} \\
-0.5347 \times 10^{7} \\
-0.1699 \times 10^{7} \\
-0.2890 \times 10^{6}\end{array}$ & $\begin{array}{r}0.5522 \times 10^{7} \\
-0.3146 \times 10^{7} \\
-0.7776 \times 10^{6} \\
-0.4832 \times 10^{5}\end{array}$ & 1.04 \\
\hline 4 & $\begin{array}{l}-0.7425 \times 10^{7} \pm 0.2743 \times 10^{1} \\
-0.6893 \times 10^{6} \pm 0.7173 \times 10^{7} \\
-0.1545 \times 10^{7} \\
-0.1353 \times 10^{6} \\
-0.5922 \times 10^{6}\end{array}$ & $\begin{array}{l}0.6010 \times 10^{6} \pm j 0.1758 \times 10^{7} \\
-0.1392 \times 10^{4} \pm j 0.1423 \times 10^{4} \\
-0.8237 \times 10^{6} \\
-0.2896 \times 10^{5} \\
-0.3183 \times 10^{6}\end{array}$ & 1.00 \\
\hline 5 & $\begin{array}{l}-0.3909 \times 10^{5} \pm j 0.4557 \times 10^{5} \\
-0.4767 \times 10^{4} \\
-0.2025 \times 10^{5} \\
-0.2885 \times 10^{5}\end{array}$ & $\begin{array}{l}-0.2218 \times 10^{5} \pm j 0.1827 \times 10^{4} \\
-0.2079 \times 10^{4} \\
-0.1171 \times 10^{6} \\
-0.1663 \times 10^{6}\end{array}$ & 1.14 \\
\hline 6 & $\begin{array}{l}-0.3986 \times 10^{6} \pm 0.3741 \times 10^{6} \\
-0.7704 \times 10^{6} \pm 0.1265 \times 10^{7} \\
-0.2096 \times 10^{7} \\
-0.1516 \times 10^{6}\end{array}$ & $\begin{array}{l}0.1419 \times 10^{5} \pm j 0.3936 \times 10^{5} \\
0.5875 \times 10^{5} \pm j 0.3713 \times 10^{5} \\
0.1861 \times 10^{6} \\
-0.3315 \times 10^{6}\end{array}$ & 1.18 \\
\hline
\end{tabular}
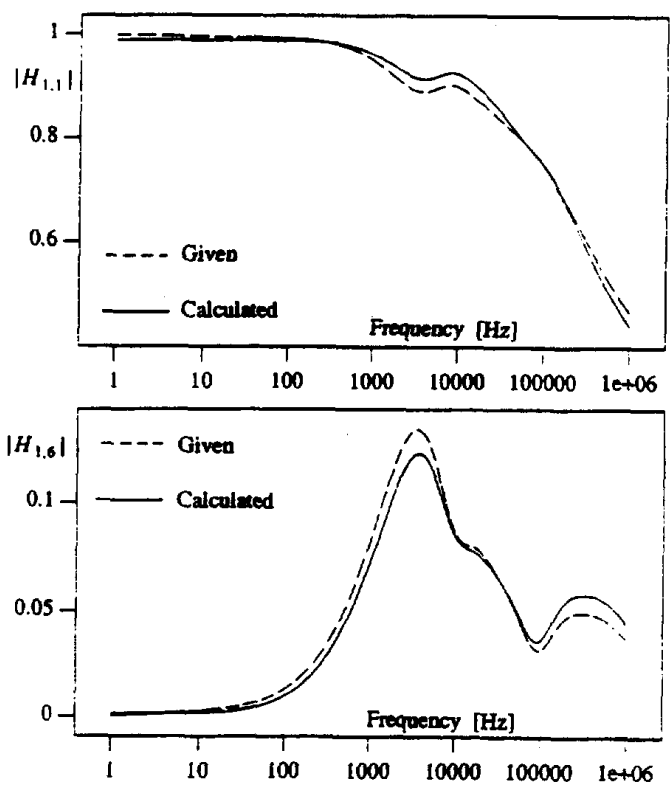

Figure 2 Comparison of the given and calculated frequency responses for $H_{1.1}$ and $H_{1.6}$. 
Fourier transforms which can be analytical if the corresponding transfer function is first approximated by a rational function. The latter is the approach used at present, in conjunction with recursive convolutions [3]-[5]. Alternatively, transfer function relations can be represented by state equations in the time domain (see Appendix of [3]), which can be integrated in the same way as the ODEs for the rest of the system.

The difficulty that arises with both recursive convolutions and state equations is related to the coupling between the traveling waves propagating along the wires of the transmission lines. Decoupling has been achieved by modal decomposition of the phase variables ( $V$ and I) but the corresponding transformation matrices $T\left(T_{V}\right.$ and $\left.T_{l}\right)$ are, in general, frequency dependent. Since the calculations are performed in the time domain, it is difficult to take this frequency dependence into account. In most cases it has been found acceptable to use a constant value $T_{0}\left(T_{V_{0}}, T_{10}\right)$ for the transformation matrix $T$, chosen at some representative frequency. The resultant accuracy is generally good in the case of single circuit overhead lines, in which case the variation of $T$ with frequency is either small or does not affect significantly the results. However, in the case of double or multiple circuit lines and cables, the frequency dependence of the modal decompositions may become more significant, in which cases, direct phase domain calculations could be expected to lead to more accurate solutions.

In this section, the conventional modal approach is reviewed, and the adequacy of the diagonalization with constant transformation matrices for various line arrangements is discussed. Finally, the developed general methodology is applied to obtain the state equation approximation of transmission line transfer matrices directly in the phase domain. One immediate consequence of the approach is that modes and modal decompositions are completely eliminated. Thus the "new" approsch becomes conceptually simpler, as simple in fact as the pre-modal analysis used to be.

\section{Computation Based on Modal Decomposition}

The computation of transients on transmission lines using the traveling wave approach is based, in present practice, on two main steps:

- Computation of the propagation effects of a (current or voltage) wave along the line from one end to the other.

- Calculation of the voltages and currents at each end of the line. These two steps involve the use of frequency domain terminal relations of the form (1), i.e.,

$$
\mathbf{y}=\mathbf{H u}
$$

where $\mathbf{H}$ is a transfer matrix while $\mathbf{u}$ and $\mathbf{y}$ are the input and outpu vectors. In relation with the wave propagation step, $\mathbf{H}=\mathbf{H}_{P}\left(\mathbf{H}_{P_{V}}\right.$ or $H_{P_{l}}$ ) is the propagation transfer matrix defined as

$$
\mathbf{H}_{P}=\mathbf{T}_{l} \mathbf{H}_{P_{m}} \mathbf{T}_{l}^{-1} \quad \mathbf{H}_{P_{m}}=\operatorname{diag}\left\{e^{-\gamma_{i l}^{l}}\right\}
$$

Here $\gamma_{l}$ are the modal propagation constants, and $T_{1}$ is the eigenvector matrix of $\mathbf{Y Z}$, where $\mathbf{Y}$ and $\mathrm{Z}$ are the line admittance and impedance matrices.

The terminal voltages and currents are calculated by using a Norton equivalent which relates an input voltage vector to an output current vector with $\mathbf{H}=\mathbf{H}_{\boldsymbol{r}}=\mathbf{Y}_{C}$, the characteristic admittance matrix:

$$
\mathrm{H}_{Y}=\mathrm{T}_{\boldsymbol{l}} \mathrm{H}_{Y_{m}} \mathrm{~T}_{\boldsymbol{V}}^{-1} \quad \mathrm{H}_{\mathrm{Ym}_{\mathrm{m}}}=\operatorname{diag}\left(Y_{C, i}\right)
$$

$\mathbf{T}_{Y}$ is the voltage transformation matrix. $\mathbf{T}_{V}$ and $\mathbf{T}_{1}$ are related by $\mathbf{T}_{v}^{f}=\mathbf{T}_{f}^{-1}$. It should be also noted that $\mathbf{H}_{p}$ is proper, while $\mathbf{H}_{r}$ is strictly proper.

The computations at the terminals are performed in the phase domain. Along the line, because of the inter-phase coupling, they are performed in the modal domain. The modal transfer matrices $\mathrm{H}_{\mathrm{P}_{m}}$ and $\mathbf{H}_{Y_{m}}$ have the advantage of being diagonal if the transformation matrices are taken at the same frequency $\omega$ as the respective transfer matrices. If not, the results are only approximate. In any case, at the two ends of the line, the currents and voltages have to be permanently converted, back and forth, from the phase to the modal domain.

\section{Adequacy of Diagonalization}

with Constant Transformation Matrices

As mentioned before, the accuracy of the modal approach depends on the effectiveness of the diagonalization over the considered frequency range. For balanced line arrangements, real constant transformations can be used to diagonalize the line transfer matrices independently of the frequency [2]. For general line configurations, fixed frequency modal transformations are used, in which case the adequacy of the diagonalization depends on the appropriate selection of the transformation frequency [14],[15].

Suppose that the given transfer matrix $\mathbf{H}$ is diagonalized over the chosen frequency range by using the transformation defined in (4). $H_{m}$ is diagonal only for the selected trensformation frequency $\omega_{b}$. At frequency values other than $\omega_{0}$ the off-diagonal elements of $\mathbf{H}_{\mathbf{r}}$ are not zero. Let the "quasi-diagonal" $\mathbf{H}_{m}$ matrix be decomposed into two matrices corresponding to its diagonal and non-diagonal parts:

$$
\mathbf{H}_{m}=\mathbf{H}_{d}+\mathbf{H}_{n}
$$

In the conventional modal approach, rational functions are fitted to the diagonal elements of $\mathbf{H}_{\text {m. }}$ The inverse transformation yields the phase domain transfer marrix, $\mathbf{H}$, which differs from the given data, because of the non-diagonality. The difference between the given transfer matrix and the one obtained by modal decomposition and back transformation can be written, taking (4) and (29) into sccount, as

$$
\mathbf{H}-\hat{\mathbf{H}}=\mathbf{T}, \mathbf{H}_{\mathrm{a}} \mathbf{T}_{\text {" }}^{-1}
$$

We define the Non-Diagonality Index as a measure of the approximation error due to the non-diagonality:

$$
\mathrm{NDI}=100 \frac{\left\|\mathrm{T}_{,} \mathrm{H}_{\mathrm{n}} \mathrm{T}_{u}^{-1}\right\|}{\left\|\mathrm{H}_{d}\right\|}
$$

In other words, NDI gives the percent error that would arise in a modal approximation if a fixed frequency transformation is used. Figure 3 shows the variation of the maximum NDI reached for the transmission lines described in Appendix B, over the frequency range from $10 \mathrm{~Hz}$ to $1 \mathrm{MHz}$, when the transformation frequency is varied within this range. Comparison of Figures 3 a and $b$ shows that the non-diagonality is higher for the propagation matrix than for the characteristic admittance matrix. The worst case arises for the propagation matrix of Line-2, where the NDI may be as high as $70 \%$, if the transformation frequency is not chosen appropriately. However, the NDI can be minimized by a particular selection of the transformation frequency. The best diagonalization is obtained at different transformation frequencies for each line.
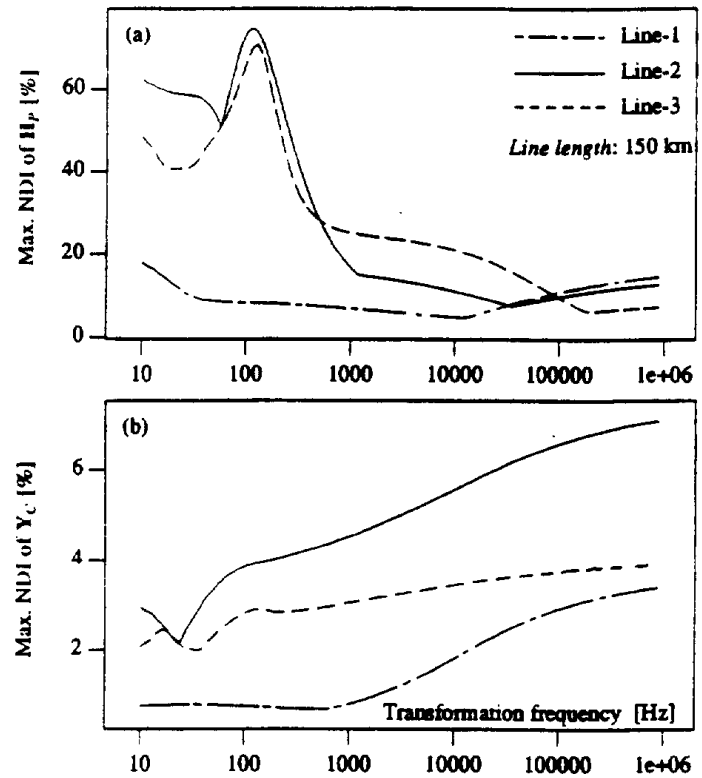

Figure 3 Variation of the maximum NDI as a function of the transformation frequency. 
In Figure 4, the variation of the NDI versus frequency is plotted for Line-2, by using the optimum transformation frequency of 34 $\mathrm{kHz}$. In the low frequency range, the NDI of $\mathbf{Y}_{c}$ is bigger than the NDI of $H_{p}$. In the frequency range above a few hundred $\mathrm{Hz}, \mathrm{H}_{p}$ has always bigger NDI, which increases at higher frequencies.

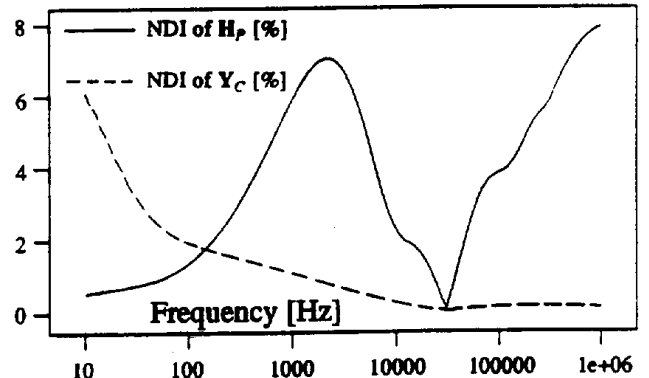

Figure 4 Variation of the NDI with frequency for Line-2 for $f_{0}=34 \mathrm{kHz}$.

NDI has also been examined in terms of the line length. This parameter appears only in the propagation matrix, therefore it affects only the NDI of $\mathbf{H}_{P}$. Table 2 shows the optimum transformation frequencies and the corresponding maximum NDI values for different lengths of Line- 2 .

Table 2 Optimum transformation frequencies for different line lengths

\begin{tabular}{|c|c|c|c|}
\hline Length $(\mathrm{cm})$ & Optimum NDI (\%) & $f_{0}(\mathrm{kHz})$ & Length $\times f_{0}$ \\
\hline 150 & 7.50 & 34.3 & 5145 \\
500 & 8.27 & 12.0 & 6000 \\
1000 & 8.37 & 6.7 & 6700 \\
\hline
\end{tabular}

From the above observations, it can be concluded that:

- The transformation frequency should be chosen appropriately to minimize the non-diagonality over the whole frequency range.

- The optimum transformation frequency depends on the geometry, wire arrangements, and on the length of the line. It varies approximately in inverse proportion to the length of the line in the example of Table 2.

- The maximum NDI obtained with the optimum transformation frequency is also a function of the line configuration and dimensions.

Consequently, although the selection of the transformation frequency is very important for the accuracy of the conventional modal approach it is not possible to state a precise, general rule for selection of the optimum transformation frequency. Even with the optimum transformation, an error in the order of $8 \%$ is introduced only as a result of the non-diagonality. The use of the direct phase domain approach eliminates these difficulties, since the non-diagonal elements of the transfer marrices are fitted by the iterative improvement process.

\section{State Equation Approximation}

of Transmission Line Transfer Matrices

In the traveling wave approach currently used in EMTP studies, the transient behavior of a transmission line is described by the propagation matrix $\mathbf{H}_{P}$ and the characteristic admittance marrix $\mathbf{H}_{Y}\left(=\mathbf{Y}_{C}\right)$ The methodology proposed in this paper can be applied for the state equation approximation of these transfer matrices directly in the phase domain.

State Equation Approximation of the propagation matrix

The propagation of a current wave along a transmission line is defined by the equation

$$
\mathbf{I}^{\prime}=-\mathbf{H}_{\boldsymbol{P}} \mathbf{I}_{\text {far }}{ }^{\prime \prime}
$$

where $H_{p}$ is defined as in (27). $I^{\prime}$ is the incident current, and $I_{p a r}{ }^{\prime \prime}$ is the current reflected at the far end. A similar expression can be given for a voltage wave as well. The propagation equation involves inherently the propagation delay

$$
\tau=\frac{l}{c}
$$

where $l$ is the length of the line and $c$ is the light velocity. In our approach the delay is taken into sccount in the time domain formulation. therefore, its effect in the frequency domain can be compensated by decomposing $H_{P}$ into two factors, as shown in [4], resulting in

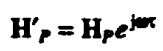

By applying the methodology presented in the first part of this paper, the state equations can be obtained directly in the phase domain as:

$$
\begin{aligned}
& \dot{\mathbf{x}}_{p}(t)=A_{p} x_{p}(t)+B_{p} I_{p_{p}}{ }^{\prime \prime}(t-\tau) \\
& I^{\prime}(t)=C_{p} x_{p}(t)
\end{aligned}
$$

The input and output variables of (35) are the reflected and incident currents defined in the phase domain.

State equation approximation of the characteristic admittance matrix The voltages and currents at the line ends are related, as shown below, by the terminal equation:

$$
I_{Y}=H_{Y} V
$$

$\mathbf{H}_{Y}$ is the characteristic admittance matrix defined in (28). The characteristic admittance of a transmission line tends to an asymprotic value at infinite frequency, i.e. $\mathbf{H}_{\gamma}$ is proper. The state equation approximation presented in this paper is developed for strictly proper systems. Therefore, $\mathbf{H}_{Y}$ must be decomposed into two parts,

$$
I_{Y}=\left(H_{Y}^{\prime}+D_{Y}\right) V
$$

where, $H_{Y}^{\prime}$ is a strictly proper transfer matrix, and $D_{Y}$ is the constant matrix determined with the limit values of the elements of $\mathbf{H}_{Y}$ for $\omega \rightarrow \infty$. The state equation approximation performed for $\mathbf{H}_{Y}^{\prime}$ yields the parameter matrices $A_{Y}, B_{Y}$, and $C_{Y}$. The state equations corresponding to (36) can then be written as:

$$
\begin{aligned}
& \dot{x}_{Y}(t)=A_{Y} x_{Y}(t)+B_{Y} v(t) \\
& I_{Y}(t)=C_{Y} x_{Y}(t)+D_{Y} v(t)
\end{aligned}
$$

The input-output variables of the state equations (38) are the phase domain terminal variables. The parameter matrices A, B, and $C$ in both (35) and (38) are refined at the final stage of the estimation process by the Gauss-Newton iterative improvement to assure the best match between the transfer matrix obtained from the state equations and the given frequency response.

\section{Calculation of Transients in the Phase Domain}

Trapezoidal integration of (35) yields:

$$
\begin{aligned}
& \mathbf{x}_{p}(t)=\mathbf{F}_{p}\left[\mathbf{1}_{\text {par }}{ }^{\prime \prime}(t-\tau-h)+\mathbf{1}_{\text {far }}{ }^{\prime \prime}(t-\tau)\right]+\mathbf{E}_{p} \mathbf{x}_{P}(t-h) \\
& \mathbf{I}^{\prime}(t)=\mathbf{C}_{p} \mathbf{x}_{p}(t)
\end{aligned}
$$

where $h$ is the integration step and

$$
E_{P}=\left[I-A_{P} \frac{h}{2}\right]^{-1}\left[I+A_{P} \frac{h}{2}\right] \quad F_{P}=\left[I-A_{P} \frac{h}{2}\right]^{-1} B_{P} \frac{h}{2}
$$

Using the frequency domain traveling wave relations

$$
V^{\prime}+V^{\prime \prime}=V \quad I+I^{\prime \prime}=I
$$

we obtain

$$
\mathbf{I}_{\boldsymbol{Y}}=\mathbf{2} \mathbf{I}^{\prime}-\mathbf{I}
$$

where

$$
I_{Y}=H_{Y} V \quad I^{\prime}=H_{Y} V^{\prime} \quad I^{\prime \prime}=-H_{Y} V^{\prime \prime}
$$

Substituting the time domain form of (42) into the state equation approximation of $I_{Y}=H_{r} V$ as given in (38), and using the trapezoidal integration rule, we can write 


$$
\begin{aligned}
& x_{Y}=F_{Y}[v(t)+v(t-h)]+E_{Y} x_{Y}(t-h) \\
& i_{Y}=G_{Y} v(t)+i_{Y h}
\end{aligned}
$$

Here, $E_{T}$ and $F_{Y}$ are defined similarly to (40), and

$$
\begin{aligned}
& G_{Y}=C_{Y} F_{Y}+D_{Y} \\
& I_{Y h}=C_{Y}\left[F_{Y} \nabla(t-h)+E_{Y} X_{Y}(t-h)\right]
\end{aligned}
$$

This yields the terminal equation

$$
I(t)=-G_{Y} v(t)+1_{h}
$$

where

$$
i_{h}=2 t^{\prime}(t)-C_{Y}\left[F_{Y} \nabla(t-h)+E_{T} x_{Y}(t-h)\right]
$$

The phase domain Norton equivalent circuit corresponding to (45) is shown in Fipure 5. It should be noted that $G_{\mathrm{r}}$ is an $r \times r$ admittance matrix and $f_{h}$ represents the history of the currents, determined in terms of the past values of phase domain variables.

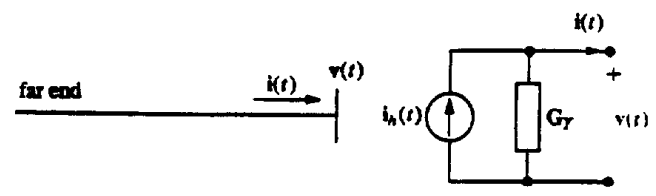

Figure 5 Phase domain Norton equivalent.

Similar terminal models can be obtained for the other end of the line. At each time step of the transient computations, the end voltages are solved from the nodal equations corresponding to the terminal connections. Line currents are evaluated by (45), and ueed to compute the refiected currents and to update all the state variables.

The calculation method described above makes it possible to compute the transients directly in the phase domain. As opposed to the classical approach, back and forth modal transfomations are not needed for the calculation of the terminal variables. Bevides the conceptual simplicity, the direct phase domain computation may provide considerable savings in computer time, particularly in the case of large networks and in computations requiring a big number of repeated simulations as, for instance, in statistical studies.

\section{RESULTS}

The direct phase domain approech was applied to compute the transients for certain switching conditions of the example tranmiesion lines described in Appendix B. Comparison with the conventional modal approach showed that both methods give similar results, in general without a significant difference. A reladively larger difference was obtained for Line-2, which also appeared to be the worst case in the NDI study. The step responses corresponding to the simultaneous energization of the six line conductors obtained by the modal and phase domain apprasches are shown in Figure 6. The difference observed is in fact negligible for most practical problems. This is a consequence of dominant diagonal elements of both $\mathbf{H}_{P}$ and $\mathbf{H}_{Y}$ matrices (see for example Figure 2), due to the large distances between conductors. It hows in the mean time that the modal approach which has been used in conventional studies provides satisfactory accuracy for practical overhead line configurations. Certainly, available field test results, used to validate the modal approach, show significantly larger differences with respect to either method, due to nomal experimental errors, than the differences between the two approaches.

Clearly, the concems often raised in relation to the frequency dependence of the transformation matrices, particularly in the case of double circuit lines, and also regarding the non-diagonality of the obtained modal transfer matrices, examined in this study using a Non-Diagonality Index (NDI), have resulted in an anticlimax in the very reassuring fact that in the time domain the modal approach is accurate in the case of overhead transmission lines.

The transients resulting from the energization of phase-a from a sinusoidal source are shown in Figure 7 as another example.

In both cases, the receiving end voltages depend only on the propagation matrix $H_{p}$ which has been shown. in conjunction with Figure 2, to be accurate to within approximately $1 \%$ with respect to
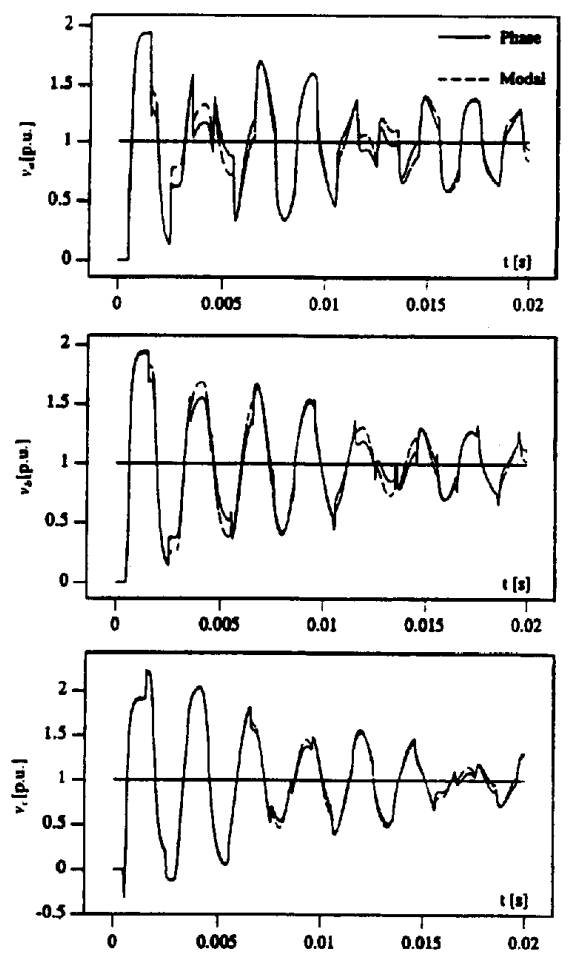

Fugure 6 Line-2. $150 \mathrm{~km}$. All a witctes closed $a t=0$. mit step response.
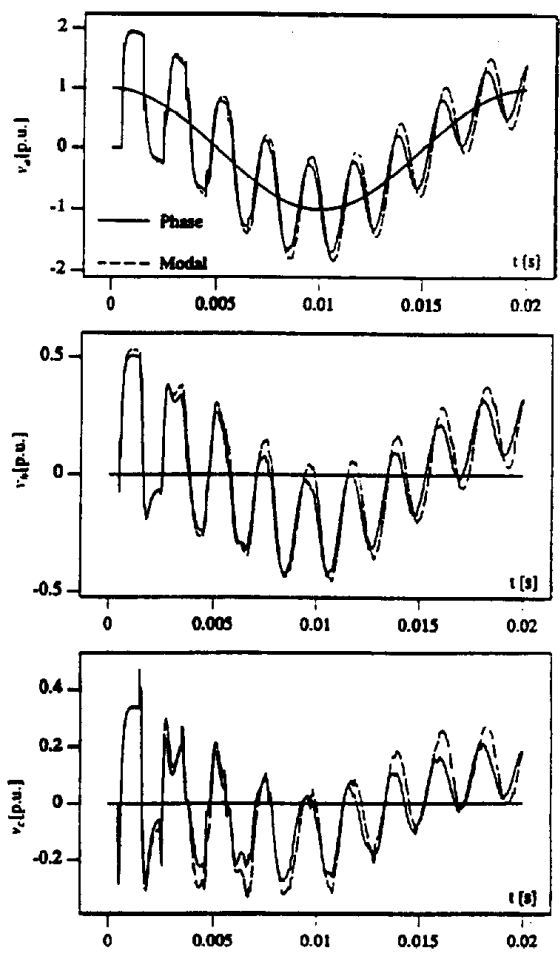

Figure 7 Transieas of single phase energization of Line-2 
the original, analytically calculated transfer matrix $\mathbf{H}_{P}$. This then is the expected magnitude of the error in the resulting time domain simulations with the phase domain approach in Figures 6 and 7. Therefore, in a practical sense, the latter may be considered as reference for assessing the accuracy of the modal approach.

The simulation examples presented above were performed with an integration step $h=0.025 \mathrm{~ms}$, which corresponds to $1 / 20$ of the travel time for a $150 \mathrm{~km}$ line. The CPU time for 800 steps of direct phase domain computations was $3.04 \mathrm{~s}$. When the same calculations were repeated using the modal state equations (7), and performing the modal transformations at each step to convert to the phase domain, the CPU time increased to 3.56 s, i.e. $17 \%$ more computer time was used. It should also be noted that the powerful approximation process used here made it possible to represent the line with state equations of smalier order, which already reduced the CPU time. Specifically, Table 1 has shown that good fiting for six modes over a wide frequency range has been achieved with only 30 poles, which is well below half of the mumber of real-only poles that would at present be required for equivalent conditions [5]. Consequently, the savings with the new, phase domain approsch can be very significant.

It is recognized that the results presented above are implementation dependent. In particular, the highly optimized features of the existing modal approach are not reflected in our comparisons. As already mentioned, for overhead transmission lines the modal aporoach is, in general, very satisfactory. Therefore, we see the main merit of the phase domain methodology in its conceptual generality and possibility of application to more difficult cases, such as cables, rather than in its features of accuracy and computational efficiency.

\section{CONCLUSIONS}

A general and widely applicable methodology is presented in this paper for the state equation approximation of multiple input-output linear systems from frequency domain data. The multiple input-output estimation is reduced by modal transformation to a scalar complex fiting problem, which is solved by a linear least squares approach featuring a constrained iterative improvement process. The final state equation approximation in the original coordinates is achieved by an iterative refinement process termed Gauss-Newton refinement. Since the scalar estimation method used is not limited to real poles only, an accurate fitting can be achieved with a smaller number of complex poles. The proposed approach makes it possible to perform time domain simulations directly in terms of the actual input-output variables, eliminating the need for modal transformations. Besides the conceptual simplicity, the new methodology provides considerable economy in the computation time especially in the case of large scale analysis and/or repetitive simulations using the same model. In the case of components with significant frequency dependence, the direct phase domain approach does not have the disadvantages due to a fixed frequency transformation, since the final approximation is improved to fit the originally given frequency domain data. We emphasize that the phase domain transfer matrix $\mathbf{H}$ is, in general, not diagonalizable with constant transformation matrices, despite the existence of a state equation realization with real coefficient matrices.

A particular application of the developed methodology is shown for the analysis of transmission line switching transients. Comparison of the results obtained with approaches based on mixed modal and phase domain calculations for different double circuit three phase line configurations have shown that both methods provide satisfactory accuracy. The computation time of the direct method appears how. ever to be less than for the modal approach currently used in EMTP calculations. Therefore, the new method could be considered a useful alternative for the computation of transmission line transients.

The direct state equation approximation method can be applied to many other problems in power engineering, including the analysis of transients in three phase cables and transformers. The frequency dependence of these components is known to be very significant. Therefore, the accuracy of the computations is expected to be improved by using the direct approach. The extension of the methodology to diverse power system components will be covered in forthcoming papers.

\section{ACKNOWLEDGEMENTS}

Financial support by the Natural Sciences and Engineering Research Council of Canada is gratefully acknowledged. The first author wishes to express his gratitude to Karadeniz Technical University, Turkey, for his sabbatical leave at the University of Toronto.

\section{REFERENCES}

[1] H.W. Dommel, "Digital Computer Solution of Electromagnetic Transients in Single- and Multiphase Networks" IEEE Trms. On Power Apparaus and Sysems, Vol. PAS-88 No.4, pp. 388-399, April 1969.

[2] H.W. Dommel and W.S. Meyer, "Computation of Electromagnetic Transien.

[3] A. Semlyen and A. Dabuleanu "Fan and Accurne Switching Transient Calculations on Transmignion Lines With Ground Retum Using Recursive Convolutions" IEEE Trans on Power
Vol. PAS-94, pp. 561-571, March/April 1975.

[4] A. Semlyen "Contributions to the Theory of Calculation of Electromagnetic Transients on Transmission Lines with Frequency Dependent Parameters" IIEE Trans. on Power

[5] J.R. Marti "Accurate Modeling of Frequency-Dependent Transmission Lines in Electromagnetic Trongient Simulations". IEEE Trans. on Power Apparatus and Sysuems, Vol. PAS-101, pp. 147-157, January 1982.

[6] P.I. Fergestad and T. Henriksen, "Inductances for the Calculation of Aransient Oncillations in Traniformers" IEEE Trans. on Power 1974.

[7] F. de Leon and A. Semlyen, "Reduced Order Model for Transformer 369, January 1992 .

[8] A.O. Soysal, "A Method for Wide Frequency Range Modeling of Power Transformers and Rotnting Machines" Proa

[9] A.O. Soyeal and A. Semlyen. "Prectical Transfer Function Estimation and Is Application to Wide Frequency Range Representation of
Transformers", IEEEPES 92 Summer Meeting, Paper No. 92 SM
$358-2-P W R D$

[10] A. Morched, L. Marti, and J. Otrevangers "A High Frequency Transformer Model for the EMTP". IEEE PES/92 Summer Meaing. Paper No. 92 SM 359-0.PWRD.

[11] A.S. Morched, J.H. Otuevangen, and L. Marti "Multi-Pont Frequency Dependent Network Equivilents for the EMTP" ", IFEE/PES Summer Meeting, Paper No. 92 SM 461-4-PWRD.

[12] T, Kailath, Linear Syetems, Chapter 6, Prentice-Hall, Inc., Englewood N., 1980.

[13] L. Marti, "Simulation of Transients in Underground Cables With Frequency-Dependem Modal Transformution Matrices". IFEE Trans. on Power Delivery, Vol. 3, No. 3. pp. 1099-1110, July 1988.

[14] P.C. Magnusson, "Traveling Wrves on Multi-Conductor Open-Wire Lines - A Numerical Survey of the Effects of Frequency Dependence of Modal Composition" IEEE Tnun. on Power Apparatus and Systems. Vol. PAS-92, pp. $999-1008,1973$.

[15] R.G. Wasley and S. Selvavinyyagamocrthy, "Approximate FrequencyResponse Values for Truminision Line Tr

[16] J.M. Ontege and W.C Rheinbold, lterative Solution of Nonlinear Equations in Several Variables , Chapter 7.5, Academic Press, New York, Landon, 1970.

[17] EPRI, Transmission Line Reference Book $345 \mathrm{kV}$ and Above, Electric

\section{APPENDICES}

Appendix A. State Equation Formulation With Real Parameters Assume that the state equations have been obtained with complex parameters as

$$
\begin{aligned}
& \dot{\mathbf{x}}(t)=\mathbf{\Lambda} \mathbf{x}(t)+\overline{\mathrm{B}} \mathbf{u}(t) \\
& \mathbf{y}(t)=\overline{\mathbf{C}} \mathbf{x}(t)
\end{aligned}
$$

where,

$$
\begin{gathered}
\Lambda=\operatorname{diag}\left\{\mathbf{A}_{k}\right\} \quad \overline{\mathbf{B}}=\operatorname{col}\left\{\overline{\mathbf{B}}_{k}\right\} \quad \overline{\mathbf{C}}=\operatorname{row}\left\{\overline{\mathbf{C}}_{k}\right\} \\
\boldsymbol{\Lambda}_{k}=\left[\begin{array}{cc}
\lambda_{k} & 0 \\
0 & \lambda_{k}^{*}
\end{array}\right] \overline{\mathbf{B}}_{k}=\left[\begin{array}{ccc}
\bar{b}_{i, 1} & \cdots & \bar{b}_{i, r} \\
\bar{b}_{i+1,1} & \cdots & \bar{b}_{i+1, r}
\end{array}\right] \quad \overline{\mathbf{C}}_{k}=\left[\begin{array}{cc}
\bar{c}_{1, i} & \bar{c}_{1, i+1} \\
\vdots & \vdots \\
\bar{c}_{r, i} & \bar{c}_{r, i+1}
\end{array}\right]( \\
(k=1, \ldots, n / 2 \quad i=2 k-1)
\end{gathered}
$$

The parameters $\lambda, \bar{b}$, and $\bar{c}$ are complex numbers defined as

$$
\lambda=\lambda^{\prime}+\mathrm{j} \lambda^{\prime \prime} \quad \bar{b}=b^{\prime}+\mathrm{j} b^{\prime \prime} \quad \bar{c}=c^{\prime}+\mathrm{j} c^{\prime \prime}
$$

Various equivalent formulations can be found to obtain state equations with real parameters leading to the same transfer matrix as ( $A$ 1). The one used in this paper is of the standard form (21), with 


$$
\begin{gathered}
A=\operatorname{diag}\left\{A_{k}\right\} \quad B=\operatorname{col}\left\{B_{k}\right\} \quad C=\operatorname{row}\left\{C_{k}\right\} \\
A_{k}=\left[\begin{array}{cc}
\lambda_{k}^{\prime} & \lambda_{k}^{\prime \prime} \\
-\lambda_{k^{\prime \prime}} & \lambda_{k^{\prime}}^{\prime}
\end{array}\right] \quad \mathbf{B}_{k}=\left[\begin{array}{ccc}
b_{i, 1} & \cdots & b_{i, r} \\
b_{i+1,1} & \cdots & b_{i+1, r}
\end{array}\right] \quad C_{k}=\left[\begin{array}{cc}
c_{1, i} & c_{1, i+1} \\
\vdots & \vdots \\
c_{r, i} & c_{r, i+1}
\end{array}\right]
\end{gathered}
$$

The parameters in (A-6) are obtained in terms of the real variables $u$ and $v$ defined as

$$
\begin{array}{rlrl}
u_{i, j}+j v_{i, j} & =\bar{c}_{i, k} \bar{b}_{k, j} \quad(j=1, \ldots, r & k=1, \ldots, n / 2 \quad i=2 k-1) \\
b_{i, j} & =u_{1, j}+v_{1, j} & b_{i+1, j} & =u_{1, j}-v_{1, j} \\
c_{j, i} & =P u_{j, 1}-Q v_{j, 1} & c_{j, i+1} & =P v_{j, 1}+Q u_{j, 1}
\end{array}
$$

where

$$
P=\frac{u_{1,1}+v_{1,1}}{u_{1,1}^{2}+v_{1,1}^{2}} \quad Q=\frac{u_{1,1}-v_{1,1}}{u_{1,1}^{2}+v_{1,1}^{2}}
$$

The real parameters have been normalized so that the first row of the C matrix is unity.

Appendix B. Description of the Example Transmission Lines The geometric dimensions and wire arrangements of the transmission lines used in the presented examples are given below. All transmission line configurations are taken from [17].

The conductor dimensions are the same for all lines:

- Phase conductors. Bundle of two conductors, 1.75" diameter, $18^{\prime \prime}$ spacing

- Ground wires: 9/16" diameter AW

The wire arrangements and dimensions of the example transmission lines are shown in Figure B-1.

A. Oguz Soysal (M'90) was bom in Turkey, in 1951. He received the B.Sc, M.Sc. and Ph.D degrees from Istanbul Technical University. In 1983 he joined ESAS Electrical Industry-power transformer factory as R\&D engineer. In 1986 he was appointed Assistant Professor at Karadeniz Technical University, Turkey. At present, Dr. Soysal is with the same institution as Associate Professor and is spending his sabbatical leave at the University of Toronto. His research areas include analysis and control of power systems, power transmission and distribution, study of electromagnetic transients and system identification.

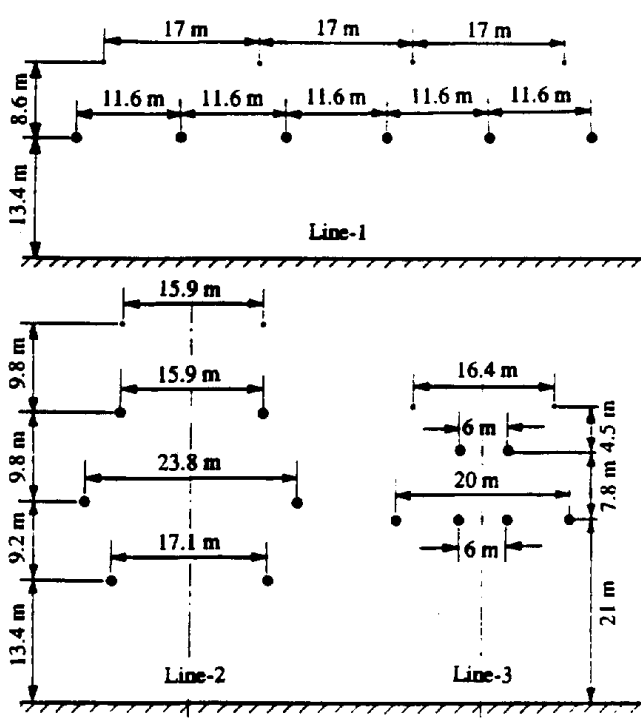

Figure B-1 Line configunioss weed in the amentical exmmples.

Adam Semlyen (F'88) was bom and educated in Rumania where he obtained a Dipl. Ing. degree and his Ph.D. He started his career with an electric power utility and held an academic position at the Polytechnic Instiute of Timisoara, Rumania. In 1969 he joined the University of Toronto where he is a professor in the Department of Electrical Engineering, emeritus since 1988. His research interests include the steady state and dynamic andysis of power systems, electromagnetic transients, and power system optimization. 